\section{Síndrome de Meckel-Gruber en un lactante menor con sobrevida prolongada. Reporte de Caso}

Ramírez-Izcoa $A E^{1}$, Sánchez-Sierra $L^{1}$, Alvarenga-Calidonio $\mathrm{RH}^{2}$, VarelaGonzález D ${ }^{3}$

Resumen

El síndrome de Meckel-Gruber es una ciliopatía debida a la disfunción del cilio celular; un trastorno autosómico recesivo letal que se caracteriza por una triada clásica de manifestaciones: displasia renal quística bilateral, meningoencefalocele occipital y polidactilia postaxial. Se presenta el caso de una lactante que al nacer presentó la triada del síndrome de Meckel-Gruber y otras alteraciones, entre las que destacó la aplasia bilateral del nervio óptico. A las 72 horas de vida fue sometida a exéresis de meningoencefalocele occipital con evolución satisfactoria, continuó con tratamiento por infecciones urinarias a repetición debido a displasia quística renal, también padeció infecciones respiratorias y falleció a los 11 meses de vida. Pese a la alta mortalidad del síndrome de Meckel-Gruber en algunos casos, poco frecuentes como éste, la sobrevida de extiende, por lo que es necesario un tratamiento multidisciplinario para tratar las complicaciones.

PALABRAS CLAVE: meningoencefalocele, polidactilia, ciliopatía, síndrome de Meckel-Gruber.

Acta Pediatr Mex. 2018 Jan;39(1):33-41.

\section{Gruber Meckel Syndrome in a young infant with prolonged survival. Case Report.}

Ramírez-Izcoa $A E^{1}$, Sánchez-Sierra $L^{1}$, Alvarenga-Calidonio $\mathrm{RH}^{2}$, VarelaGonzález D ${ }^{3}$

\begin{abstract}
Meckel-Gruber Syndrome is a ciliopathy caused by dysfunction of primary cilia, is a lethal autosomal recessive disorder that is diagnosed by the presence of a classical triad manifestations: bilateral cystic renal dysplasia, occipital meningoencephalocele and postaxial polydactyly. We present a case of a female infant, who at birth presented the triad Syndrome Meckel Gruber and other conditions, among which highlighted the bilateral aplasia of the optic nerve. The patient underwent exceresis of occipital meningoencephalocele at 72 hours of life, with satisfactory evolution; she continued with treatment for urinary secondary to the renal cystic dysplasia infections, also pre-
\end{abstract}

${ }^{1}$ Doctor en medicina y cirugía, Facultad de Ciencias Médicas.

${ }^{2}$ Pediatra y Genetista (egresado del INP), Profesor de Pediatría y Genética Médica, Hospital Escuela Universitario, UNAH. Presidente Asociación Pediátrica Hondureña.

${ }^{3}$ Pediatra Neurólogo (egresado del INP), Profesor Titular III Facultad de Ciencias Médicas. Hospital Escuela Universitario, Honduras.

Universidad Nacional Autónoma de Honduras, Honduras.

Recibido: 18 de mayo del 2016

Aceptado: 16 de marzo del 2017

Correspondencia

Alejandro Ramírez-Izcoa

alejandro_izcoa@hotmail.es

Este artículo debe citarse como

Ramírez-Izcoa AE, Sánchez-Sierra LE, AlvarengaCalidonio RH, Varela-González D. Síndrome de Meckel-Gruber en un lactante menor con sobrevida prolongada. Reporte de Caso. Acta Pediatr Mex. 2018;39(1):33-41. 
sented respiratory infections and died at 11 months. Despite the high mortality Meckel Gruber syndrome, in unusual cases like this, they have longer survival, so it is necessary to establish a multidisciplinary approach to treat complications.

KEYWORDS: meningoencephalocele; polydactylylism; ciliopathy
Correspondence

Alejandro Ramírez-Izcoa

alejandro_izcoa@hotmail.es

\section{INTRODUCCIÓN}

El síndrome de Meckel-Gruber (MGS) es una ciliopatía causada por disfunción del cilio celular. ${ }^{1-3}$ Fue descrito por primera vez en 1822. Es un trastorno autosómico recesivo letal caracterizado por una triada de manifestaciones: displasia renal quística bilateral, meningoencefalocele occipital y polidactilia postaxial. ${ }^{1-5}$ Se puede acompañar de una amplia variedad de malformaciones entre las que destacan la fisura palatina descrita por Meckel en 1822 y la fibrosis hepática descrita por Gruber en 1934. 5,6

La prevalencia estimada a nivel mundial oscila entre 1/1,300 hasta 1/140,000. Sin embargo, la población en la que se presenta con más frecuencia es en los indios Gujarati, en el oeste de la India, con 1/1,300. En Gran Bretaña, en un estudio publicado en el 2014, se reportó una prevalencia en Europa de 2,6 por cada 100,000 nacimientos. El número de casos de MGS en los registros de EUROCAT (red de registros de población para la vigilancia epidemiológica de las anomalías congénitas en Europa) reportó, entre 1990 y el 2011, 142 casos en 9 países, de los cuales 62 pertenecían a Gran Bretaña. Hay evidencia de que la prevalencia es mayor en poblaciones con mayores índices de consanguinidad como la India, Pakistán, Kuwait y otros países árabes; actualmente no hay suficientes datos epidemiológicos a nivel mundial. ${ }^{7,8}$

De acuerdo a la literatura consultada, en Latinoamérica se han reportado casos diagnosticados durante el periodo prenatal y autopsias de óbitos en: México, Cuba, Colombia y Venezuela, no se encontraron casos descritos en Honduras.

Las ciliopatías son un grupo de trastornos del desarrollo cuya expresividad fenotípica sigue siendo poco clara. ${ }^{1}$ El fenotipo de los pacientes con ciliopatías viene determinado por cuatro posibles mecanismos genéticos: mutaciones homocigóticas o doble heterocigotas, alelos múltiples, genes modificadores y oligogenicidad verdadera. ${ }^{9}$

El MGS es una enfermedad genéticamente heterogénea y se han descrito 14 genes ciliares asociados a este síndrome. ${ }^{8}$ Los genes y sus loci principalmente identificados en el MGS son: MKS1 en 17q21-q24, MKS2 en 11q13 y MKS3 en $8 \mathrm{q} 24 .{ }^{10,11}$ Se ha sugerido que las proteínas implicadas en el MGS participan en cascadas de señalización durante el desarrollo embrionario; sin embargo, los mecanismos de patogenicidad y el papel de estas proteínas durante el desarrollo están todavía en estudio. ${ }^{10}$

El MGS se considera una enfermedad letal porque un tercio de los niños afectados muere antes de nacer, el resto sobrevive como promedio 3 horas. Hasta el 2013, en una revisión realizada por Parelkar et. al, sólo se habían reportado 10 casos a nivel mundial con sobrevida prolongada, de los cuales únicamente 4 llegaron a etapa de lactantes; 3,12 en las bases de datos revisadas no se encontraron reportes de pacientes con sobrevida prolongada en Latinoamérica.

En este reporte se describe caso de una paciente con MGS y aplasia bilateral del nervio óptico, 
con sobrevida prolongada hasta los 11 meses; lo anterior hace relevante la presentación de este caso pues existen muy pocos datos publicados sobre la evolución de los nacidos vivos, debido a que la mayoría de los casos con este síndrome son terminaciones de embarazo después del diagnóstico prenatal.

\section{PRESENTACIÓN DE CASO CLÍNICO}

Niña de 11 meses de vida, hondureña, producto de la primera gesta de madre de 19 y padre de 37 años, no consanguíneos según refirieron. Durante los controles prenatales se detectaron oligohidramnios y defectos congénitos múltiples, sin reportar MGS. Parto normal, a término de 37 semanas de gestación, con Apgar de 6/7 y con datos de hipoxia cerebral, reflejo de moro incompleto. Peso al nacer de $2.7 \mathrm{~kg}$, perímetro cefálico de $31 \mathrm{~cm}$, por debajo del percentil 3 según tablas del CDC, cráneo asimétrico con frente amplia y prominente, defecto óseo y masa que protruye en la región occipital con dimensiones de $4.54 .5 \mathrm{~cm}$ correspondiente a meningoencefalocele occipital, al que se le practicó exéresis a las 72 horas de vida (Figura 1A); también tenía dos protuberancias sublinguales, una lateral derecha y otra lateral izquierda, a las que se les realizó resección quirúrgica resultando, en estudio histopatológico, hamartoma lingual bilateral (Figura 1B). Ojo derecho con fisura palpebral que permanecía cerrada y fisura palpebral izquierda pequeña, ambos globos oculares de apariencia normal, paladar íntegro, cuello corto, abdomen con masas lobuladas en ambos flancos, genitales femeninos sin alteraciones evidentes, extremidades con polidactilia postaxial en manos y pies (Figura 2).

La tomografía cerebral simple posterior a cirugía reveló dilatación de los ventrículos laterales, principalmente de los cuernos anteriores, disminución del volumen de los hemisferios cerebrales con defecto en el parénquima de la región occipital secundario al meningoencefalocele, hipotrofia del cerebelo, disgenesia del cuerpo calloso y de los ganglios basales. La resonancia magnética de control (Figura 1C) mostró dilatación severa de los ventrículos laterales y el tercer ventrículo como compensación a la disminución de volumen de los hemisferios cerebrales, con moderado ensanchamiento del espacio subaracnoideo parietal bilateral; hipoplasia del cuerpo calloso, signos de atrofia córtico-subcortical moderada generalizada; en fosa posterior adelgazamiento del tallo cerebral con ensanchamiento del IV ventrículo y cambios de hipotrofia de los hemisferios cerebelosos relacionados con meningoencefalocele ya reparado, también se apreciaba lisencefalia focal en hemisferio occipital izquierdo.

Durante su estancia se le practicó ultrasonido transfontanelar que reportó cuernos anteriores de ventrículos laterales dilatados sin imágenes patológicas intraventriculares; el resto del sistema ventricular se apreciaba sin alteración. Se realizó un ultrasonido abdominal para descartar otras malformaciones intra-abdominales que reportó: hígado, vesícula biliar, páncreas y bazo con tamaño y morfología dentro de los parámetros adecuados. Los ultrasonidos renal y de las vías urinarias mostraron aumento de volumen de ambos riñones con múltiples formaciones quísticas en todo el parénquima renal en relación con displasia renal multiquística bilateral (Figura 3).

Se efectuó un electroencefalograma, en condiciones de sueño inducido bajo sedación, con resultado anormal por la existencia de actividad de fondo asincrónica y por actividad epileptiforme en la región occipital de ambos hemisferios, de presentación independiente, de magnitud moderada, lo que fue indicativo de una disfunción córtico-subcortical generalizada y moderados signos irritativos de la región occipital de ambos hemisferios.

Se interconsultó con cardiología pediátrica donde se detectó, mediante ecocardiograma 

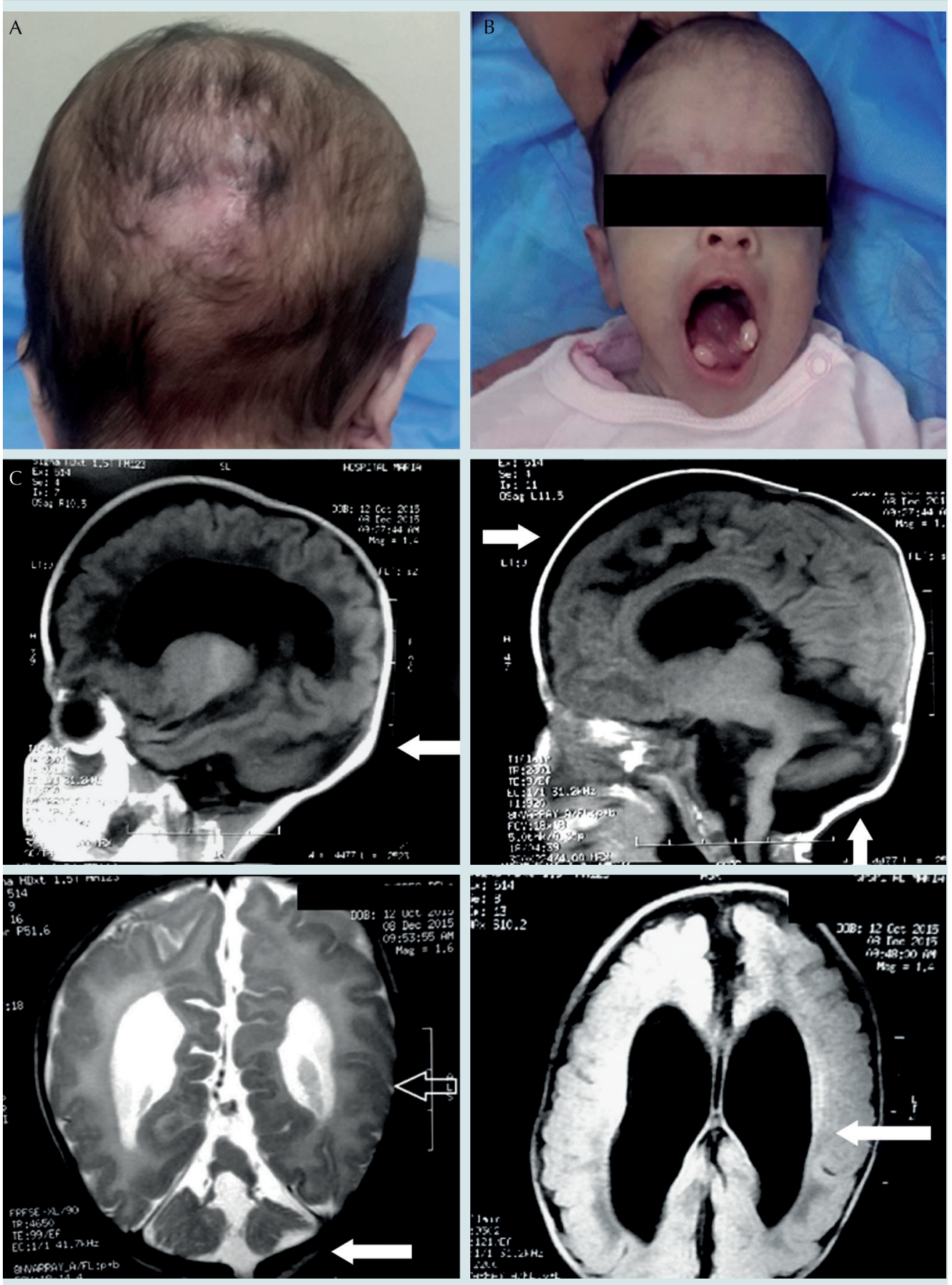

Figura 1. A) Cicatriz secundaria a exéresis de meningoencefalocele. B) Lengua con quistes, hamartoma bilateral y perímetro cefálico de $36 \mathrm{~cm}$ a los 4 meses de edad. C) Resonancia magnética, las flechas indican: área de secuelas de exéresis de meningoencefalocele occipital, dilatación de ventrículos laterales, hipoplasia del cuerpo calloso, hipotrofia en hemisferios. 

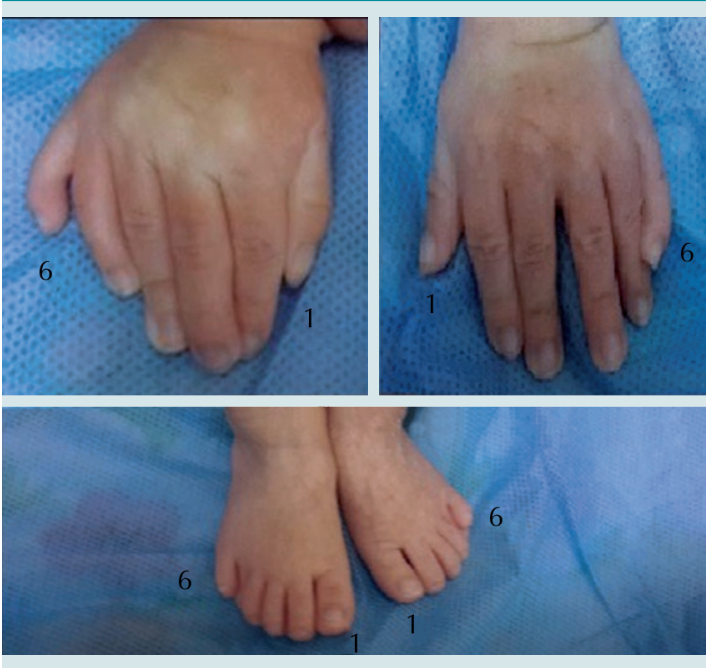

Figura 2. Polidactilia postaxial en las cuatro extremidades.

bradicardia sinusal, persistencia de foramen oval y soplo grado II/IV. Oftalmología indicó que al fondo del ojo sin dilatar la pupila (por contraindicación al uso de atropina) se observó aplasia bilateral del nervio óptico. En la última evaluación por genética, a los 4 meses de edad, la paciente tenía un perímetro cefálico de $36 \mathrm{~cm}$, ubicándose debajo del percentil 3 según las tablas del CDC, indicando una evidente microcefalia, reflejos osteotendinosos normales, reflejo de prensión palmar bilateral, sin sostén cefálico; un estudio de citogenética reportó 46,XX en 20 metafases. Los padres de la paciente recibieron consejo genético y asesoría sobre cuidados y terapias para prevenir complicaciones de la enfermedad de base. A los 11 meses de vida la paciente falleció por neumonía adquirida en la comunidad y choque séptico.

\section{DISCUSIÓN}

Las patologías asociadas a disfunción ciliar se denominan ciliopatías. Éstas obedecen a una alteración de genes implicados en la formación y señalización ciliar, que generan alteraciones en la inducción del mesodermo embrionario, por lo
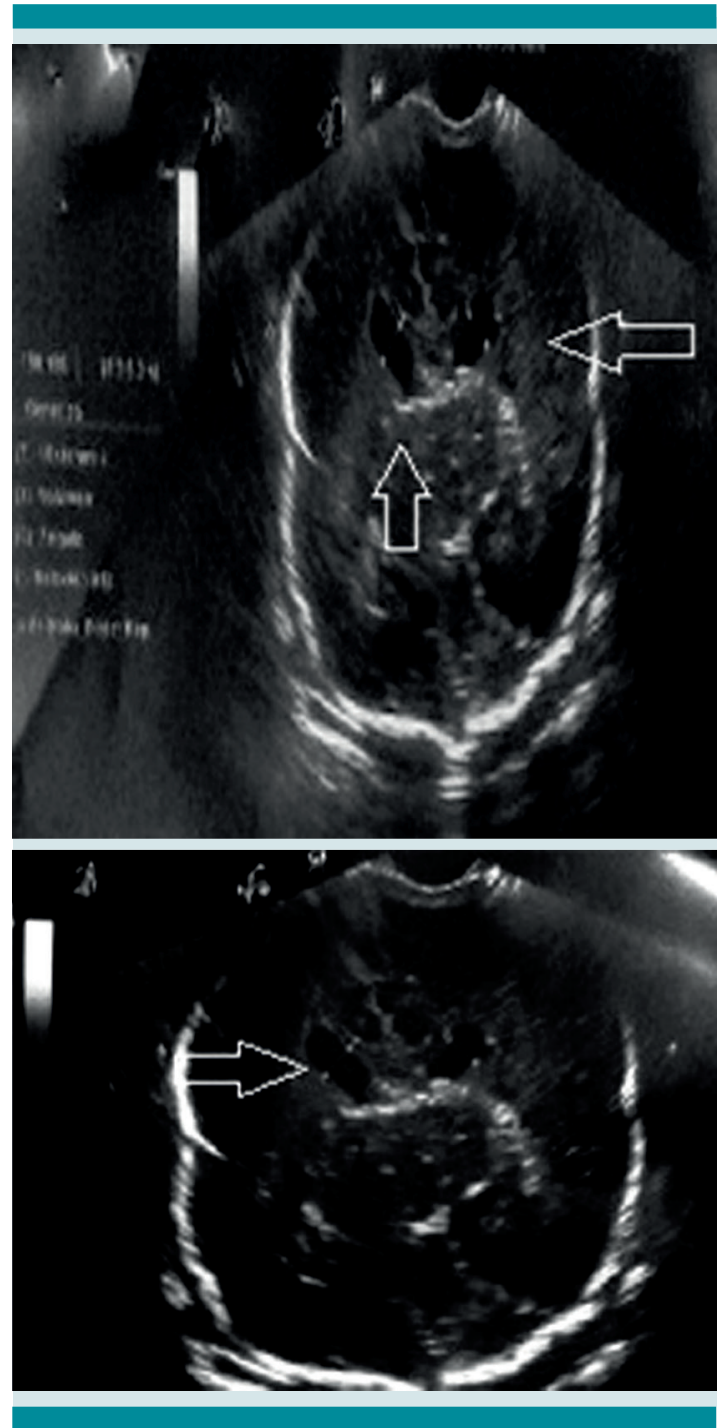

Figura 3. Ultrasonido renal. Las flechas indican múltiples quistes renales.

tanto muchas de ellas presentan manifestaciones neurológicas y diversas malformaciones como el síndrome de Bardet-Bield, Joubert y el MGS. ${ }^{9}$

Los criterios mínimos para establecer el diagnóstico clínico de MGS son la presencia de al menos dos de las tres manifestaciones principales, es decir, displasia renal quística bilateral, meningoencefalocele occipital y polidactilia. ${ }^{2,3,8}$ 
En este caso, la paciente contó con los componentes de la triada clásica, además de otras características que apoyan el diagnóstico como oligohidramnios, microcefalia, cardiopatía congénita y alteraciones del sistema nervioso central identificadas en los estudios de neuroimagen. ${ }^{13}$

Los diagnósticos diferenciales de MGS incluyen al síndrome de Bardet-Biedl, al síndrome de Smith-Lemli-Opitz y a la trisomía 13. Se descartó el diagnóstico de Bardet-Biedl por la presencia de encefalocele. El síndrome de Smith-LemliOpitz se manifiesta con disfunción hepática y colestasis, así como fisura palatina o úvula bífida, datos que tampoco se presentaron en este caso. La trisomía 13 es el principal diagnóstico diferencial del MGS, por lo que el resultado del cariotipo es indispensable para diferenciarlos; ${ }^{13-16}$ sin embargo, clínicamente la trisomía 13 muestra: paladar hendido, microoftalmia y holoprocencefalia, ${ }^{11,13}$ también ausentes en la paciente.

El meningoencefalocele occipital fue diagnosticado al nacimiento; sin embargo, éste y los componentes de la triada clásica pueden identificarse desde la etapa prenatal. ${ }^{8}$ En esta paciente no se hizo diagnóstico por ultrasonido prenatal, ya que no fue valorada en un hospital de especialidades y sólo se reportó como "defectos congénitos múltiples". En la etapa postnatal los defectos congénitos cráneo-encefálicos constituyen una malformación fácil de reconocer, así es que el encefalocele suele diagnosticarse mediante la inspección, presentando un aspecto de masa sésil o pedunculada de tejido blando de tamaño y configuración variables; ${ }^{17}$ posterior al diagnóstico en esta paciente éste fue sometido a exéresis a las 72 horas de vida. El pronóstico de la cirugía depende de la presencia o ausencia de masa encefálica dentro del saco herniario, así como de malformaciones asociadas. ${ }^{18} \mathrm{Al}$ evaluar la paciente cuatro meses después por el servicio de genética y neurología, ésta presentó retraso en el desarrollo psicomotor y no tuvo convulsiones.
Entre otros defectos comúnmente observados en el sistema nervioso central, en los pacientes con MGS, se pueden incluir: disgenesia rómbica y disgenesia del prosencéfalo, este último puede incluir disgenesia bulbo olfatorio, hipoplasia del nervio óptico, agenesia del cuerpo calloso, holoprosencefalia, ${ }^{1}$ hidrocefalia y agenesia del cerebelo. ${ }^{12}$ La paciente presentó: hipoplasia del cuerpo calloso, disminución de volumen de los hemisferios cerebrales, atrofia córtico subcortical moderada generalizada, adelgazamiento del tallo cerebral con ensanchamiento del IV ventrículo e hipotrofia de los hemisferios cerebelosos. Sumado a estos defectos, la paciente también tenía aplasia del nervio óptico bilateral, una afección poco común. En la revisión realizada por Parelkar en 2013 (Cuadro 1) no se describe a la aplasia bilateral del nervio como parte del MGS; sin embargo, ya que en la literatura y en las bases de datos sí hay registros de hipoplasia del nervio óptico ${ }^{12,13}$ podemos añadir a la descripción del síndrome esta alteración. Se propone que la aplasia bilateral del nervio óptico se debe a la relación que existe entre las ciliopatías y ciertas enfermedades monogénicas degenerativas, muchas de ellas con manifestaciones oftalmológicas y neurológicas. ${ }^{9}$

En la enfermedad poliquística renal la ciliopatía deriva del cambio estructural en la poliquistina y en la policisteína, proteínas fundamentales para la diferenciación de las células de los túbulos renales, ${ }^{9}$ este es el principal hallazgo clínico presente en el $100 \%$ de los casos. ${ }^{10} \mathrm{La}$ enfermedad quística renal se ha asociado con complicaciones como: infecciones del tracto urinario, hipertensión arterial, malignización y causa de mortalidad junto con los defectos del sistema nervioso central y enfermedades respiratorias. ${ }^{13,19}$

Algunos autores sugieren ampliar el espectro de anomalías encontradas en el MGS e incluir las malformaciones cardíacas por la frecuencia entre ambos, pues éstas se han reportado en el 
Cuadro 1. Casos reportados en la literatura con sobrevida prolongada

\begin{tabular}{|c|c|c|c|c|c|c|}
\hline Referencia & Año & $\begin{array}{l}\text { No. de } \\
\text { casos }\end{array}$ & Sexo & $\begin{array}{l}\text { Peso al } \\
\text { nacer }\end{array}$ & $\begin{array}{c}\text { Edad de } \\
\text { fallecimiento }\end{array}$ & Malformaciones \\
\hline $\begin{array}{l}\text { Ramadani, } \\
\text { et al. }\end{array}$ & 1992 & 1 & M & $2.8 \mathrm{~kg}$ & 28 meses & $\begin{array}{l}\text { Encefalocele occipital, polidactilia y paladar } \\
\text { hendido. }\end{array}$ \\
\hline $\begin{array}{l}\text { Paavola, } \\
\text { et al. }\end{array}$ & 1995 & 1 & M & $2.5 \mathrm{~kg}$ & 18 meses & Encefalocele, riñón poliquístico y polidactilia \\
\hline $\begin{array}{l}\text { Gazioglu, } \\
\text { et al. }\end{array}$ & 1998 & $1 *$ & M & $3.4 \mathrm{~kg}$ & 7 meses & $\begin{array}{l}\text { Polidactilia bilateral, hernia inguinal, disminución } \\
\text { global de reflejos neonatales e hipotonía. }\end{array}$ \\
\hline Vince, et al. & 1998 & $3 *$ & M & $3.0 \mathrm{~kg}$ & $\begin{array}{l}\text { Caso 1: } 5 \text { días } \\
\text { Caso 2: } 1 \text { hora } \\
\text { Caso 3: } 4 \text { meses }\end{array}$ & $\begin{array}{l}\text { Caso 1. Encefalocele occipital, polidactilia, paladar } \\
\text { alto arqueado. Caso 2. Encefalocele, polidactilia mas } \\
\text { otras alteraciones. Caso } 3 \text {. Encefalocele y polidacti- } \\
\text { lia. }\end{array}$ \\
\hline $\begin{array}{l}\text { De Silva, } \\
\text { et al. }\end{array}$ & 2004 & 1 & M & $2.2 \mathrm{~kg}$ & 1 día & $\begin{array}{l}\text { Encefalocele posterior, riñones poliquísticos, } \\
\text { polidactilia, holoproscencefalia. }\end{array}$ \\
\hline Walsh, et al. & 2006 & 1 & M & $3.0 \mathrm{~kg}$ & 10 días & $\begin{array}{l}\text { Meningocele occipital, malformación de Dandy- } \\
\text { Walker, riñón quístico agrandado. }\end{array}$ \\
\hline $\begin{array}{l}\text { Ramachan- } \\
\text { dran, et al. }\end{array}$ & 2006 & 1 & M & $3.1 \mathrm{~kg}$ & NR & $\begin{array}{l}\text { Meningoencefalocele occipital, fisura palatina cen- } \\
\text { tral, micrognatia, microcefalia, ausencia parcial del } \\
\text { septum nasal, cardiomegalia con soplo pansistólico } \\
\text { y criptorquidia. }\end{array}$ \\
\hline $\begin{array}{l}\text { Abdelmo- } \\
\text { neim EM } \\
\text { Kheri, et al. }\end{array}$ & 2012 & 1 & $\mathrm{~F}$ & $4.0 \mathrm{~kg}$ & 3 días & $\begin{array}{l}\text { Encefalocele occipital, polidactilia, riñones } \\
\text { poliquísticos, labio y paladar hendido. }\end{array}$ \\
\hline Este reporte & 2017 & 1 & $\mathrm{~F}$ & $2.7 \mathrm{~kg}$ & 11 meses & $\begin{array}{l}\text { Encefalocele occipital, hipoplasia de cuerpo calloso } \\
\text { y nervios ópticos, hamartomas linguales, cardiopatía, } \\
\text { polidactilia y riñones poliquísticos. }\end{array}$ \\
\hline
\end{tabular}

NR: No reportada. *Consanguinidad Presente. Modificado de Parelkar et. al $2013 .{ }^{3}$

$20 \%$ de los casos. ${ }^{11,20,21}$ La paciente de este reporte tuvo, a nivel cardíaco, bradicardia sinusal, persistencia de foramen oval y soplo grado II/IV. En lo concerniente al encefalocele occipital y la polidactilia postaxial éstos se presentan en el 90 y $80 \%$ de los pacientes, respectivamente. ${ }^{12,18}$

El MGS es letal; sin embargo, este caso presentó una sobrevida prolongada, haciéndolo un caso muy relevante ya que hay pocos datos publicados sobre cualquier tipo de nacimiento, muertes fetales y terminaciones de embarazo tras el diagnóstico prenatal. John D. Vince, en 1998, documentó por primera vez una familia de Papúa, Nueva Guinea, con tres bebes que cumplían con los criterios diagnósticos para MGS, pero los dos primeros casos no se documentaron. En 1995,
Paavola reportó otro caso compatible con MGS que murió a los 18 meses de vida. ${ }^{22}$ Hay sólo dos informes de pacientes que sobrevivieron más allá de la infancia y, de acuerdo con Ramadani, existe un reporte de un sobreviviente que murió a la edad de 28 meses. ${ }^{23}$

Las altas mortalidad y morbilidad se deben a las alteraciones renales, el hígado no funcional y la hipoplasia pulmonar; ${ }^{3}$ en este caso la paciente padecía displasia renal quística con infecciones urinarias a repetición. Los defectos de nacimiento suelen plantear un reto en el tratamiento, las medidas preventivas deben comenzar a nivel de atención primaria, para asegurar el bienestar físico y mental óptimo de las mujeres, ${ }^{2}$ así como la consejería genética a los padres, tal como se 
realizó en este caso. Los padres pueden ser asesorados acerca de la evolución, pronóstico y la recurrencia de riesgo para el siguiente embarazo que es del $25 \%$. $^{14,15}$

EI MGS puede ser diagnosticado por ultrasonografía (USG) desde el primer trimestre de embarazo, entre las 11 y 14 semanas de edad gestacional. ${ }^{6-8,24} \mathrm{El}$ estudio realizado por AlBelushi M. et al., en 2015, recomienda que el diagnóstico en el embarazo temprano no requiere la triada clásica de encefalocele, polidactilia y los riñones poliquísticos, ya que algunas de estas características no se manifiestan en las imágenes hasta mucho más tarde. ${ }^{25} \mathrm{El}$ diagnóstico prenatal de riñones poliquísticos, agrandados y bilaterales, debe alertar y ser punto de partida para una investigaciones exhaustiva en busca de malformaciones del sistema nervioso y polidactilia que completen la triada clásica. ${ }^{16}$

\section{CONCLUSIONES}

El diagnóstico del MGS puede realizarse desde el primer trimestre del embarazo, por lo que un control prenatal adecuado permite identificar de manera temprana las características de la triada clásica: displasia renal quística bilateral, meningoencefalocele occipital y polidactilia postaxial, para realizar un diagnóstico temprano y determinar la conducta a seguir con el fin de preparar las condiciones óptimas en la atención del parto y permitir mejorar, en lo posible, la calidad de vida de los pacientes.

Pese a la alta mortalidad en algunos casos poco frecuentes, como éste, la sobrevida se extiende, por lo que es necesario un tratamiento multidisciplinario para tratar las complicaciones que ponen en riesgo la vida y realizar una evaluación exhaustiva, ya que se pueden presentar alteraciones poco documentadas en otros casos como la aplasia bilateral del nervio óptico.

\section{REFERENCIAS}

1. Abdelhamed Z, Wheway G, Szymanska K, Natarajan S, Toomes C, Inglehearn C, et al. Variable expressivity of ciliopathy neurological phenotypes that encompass Meckel-Gruber syndrome and Joubert syndrome is caused by complex de-regulated ciliogenesis, Shh and Wntsignalling defects. Hum Mol Gen. 2013;22(7):1358-1372. http://hmg. oxfordjournals.org/content/22/7/1358

2. Myageri A, Grampurohit V, Rao R. Meckel Gruber Syndrome: Report of Two Cases with Review of Literature. J Family Med Prim Care. 2013;2(1):106-108 http://www.jfmpc.com/ article. asp?issn=2249-4863; year=2013; volume $=2$;issue $=1$ ;spage=106; epage=108; aulast=Myageri

3. arelkar SV, Kapadnis SP, Sanghvi BV, Joshi PB, Mundada D, Oak SN. Meckel Gruber syndrome: A rare and lethal anomaly with review of literature. J Pediatr Neurosci. 2013;8(2):154-157. http://www.pediatricneurosciences. com/article.asp?issn=1817-1745; year $=2013$; volume $=8$; is sue $=2$; spage $=154$; epage $=157$; aulast $=$ Parelkar

4. Molin A, Benoist G, Jeanne-Pasquier C, Elkartoufi N, Litzer J, Decamp M, et al. 12q21 Microdelection in a fetus with Meckel syndrome involvig CEP290/MKS4. Eur J Hum Genet. 2013;56(1):580-583. http://europepmc.org/abstract/ MED/23954617

5. Martínez-Mendel J, Sanz Asin O, Amat-Villegas I, AzconaRuiz B, Cabistany-Esque A, de-Marcos E. Sindrome de Meckel. Diagnóstico Prenatal y Diagnostico Diferencial. Prog Obstet Ginecol. 2012;55(6):269-273. http://bvsalud. org/portal/resource/en/ibc-100315

6. Panduranga C, Kangle R, Badami R, Patil PV. Meckel-Gruber syndrome: Report of two cases. J Neurosci Rural Pract. 2012;3:56-9. Disponible en: https://www.ncbi.nlm.nih. gov/pmc/articles/PMC3271618/

7. EUROCAT. European Surveillance of congenital anomalies. Italia. Disponible en: http://www.eurocat-network.eu/

8. Barisic I, Boban L, Loane M, Garne E, Wellesley D, Calzolari E. Meckel-Gruber Syndrome: a population-based study on prevalence, prenatal diagnosis, clinical features, and survival in Europe. Eur J Hum Genet. 2015;23:746-752. http://www.ncbi.nlm.nih.gov/pubmed/25182137

9. Silva E D, Pinazo-Durán M D. Ciliopatías y su relación con la oftalmología. Arch Soc Esp Oftalmol. 2013;88(5):165-167. http://apps.elsevier.es/watermark/ ctl_servlet?_f=10\&pident_articulo=90199673\&pident_ usuario $=0$ \&pcontactid $=\&$ pident_revista $=296 \&$ ty $=80 \&$ acc ion=L\&origen=zonadelectura\&web=www.elsevier.es\&lan $=$ es\&fichero=296v88n05a90199673pdf001.pdf

10. Eckmann-Scholz C, Jonat W, Zerres K, Ortiz-Brüchle N: Earliest ultrasound findings and description of splicing mutations in Meckel-Gruber Syndrome. Arch Gynecol Obstet 2012;286:917-921. Disponible en: http://link.springer. com/article/10.1007\%2Fs00404-012-2411-6

11. Borislav AA, Xiaoqing L, Chen-Chih S, Brenner DS. MeckelGruber Syndrome: Pathologic Manifestations, Minimal 
Diagnostic Criteria, and Differential Diagnosis. Archives of Pathology \& Laboratory Medicine. 2006;130(8):12361238. Disponible en: http://www.archivesofpathology.org/ doi/full/10.1043/1543-2165(2006)130[1236:MS]2.0.CO;2

12. Medina $M$ L, Saldarriaga $W$, Isaza $C$, Pachajoa H. Síndrome de Meckel con onfalocele y labio fisurado. Rev Cubana Obstet Ginecol. 2014;40(2):272-278. http://www.medigraphic. com/pdfs/revcubobsgin/cog-2014/cog142n.pdf

13. Jones, LJ, Jones MC, Del Campo M. Smith's Recognizable Patterns of Human Malformation. 7th ed. Elsevier Saunders: Philadelphia, PA, 2013.

14. Myageri A, Grampurohit V, Rao R. Meckel Gruber Syndrome: Report of Two Cases with Review of Literature. Journal of Family Medicine and Primary Care. 2013;2(1):106-108. Disponible en: https://www.ncbi.nlm.nih.gov/pmc/articles/PMC3894002/

15. Hakverdi S, Güzelmansur I, Sayar H, Arif G, Hakverdi AU, Toprakl S. Meckel-Gruber syndrome: A report of three cases. Perinat J. 2010;18:59-63. Disponible en: http:// www.perinataljournal.com/Archive/Article/20100182005

16. Chen CP. Meckel syndrome: Genetics, perinatal findings and differential diagnosis.Taiwan J Obstet Gynecol. 2007;46:9-14. Disponible en: https://www.ncbi.nlm.nih. gov/pubmed/17389183

17. Peñaloza-Valenzuela JJ, Torrico-Aponte WA, TorricoAponte ML, Peñaloza-Florez M. Encefalocele occipital gigante neonatal, a propósito de un caso. Gac Med Bol. 2011;34(2):99-101. http://www.scielo.org.bo/scielo. php?pid=S1012-29662011000200011\&script=sci_abstract

18. Dawe HR, Smith UM, Cullinane AR, Gerrelli D, Cox P, Badano JL, et al. The MeckelGruber Syndrome proteins MKS1 and meckelin interact and are required for primary cilium formation. Human Molecular Genetics.
2007;16(2):173-Disponible en: http://hmg.oxfordjournals. org/content/16/2/173.long

19. Ubetagoyena M, Areses R, Arruebarrena D. Displasia renal multiquística unilateral. Revisión de nuestra casuística. BOL. S VASCO-NAV PEDIATR 2004;37:30-32. Disponible en: http://www.svnp.es/sites/default/files/37-1-30.pdf

20. Uysal F, uysal A. Meckel-Gruber Syndrome with unilateral renal agenesis. J Coll Physicians Surg Pak. 2015;25(11):5657. http://www.ncbi.nlm.nih.gov/pubmed/25933467

21. Kurtulmuş S, Demirpençe S, Can Öztekin D, Koç A, Tavli $V$. Antenatal diagnosis of left atrial isomerism and heterotaxy syndrome in fetus with Meckel-Gruber syndrome. Turk Kardiyol Dern Ars. 2014;42(2):182-185. http://arsiv. tkd.org.tr/ /media/files/archive/documents/2014/mart/ tkda_2014_42_2_182-185.pdf

22. Blankenberg TA, Ruebner BH, Ellis WG, Bernstein J, Dimmick JE. Patología de las anomalías renales y hepáticas en el síndrome de Meckel. Am J MedGenetSupl. 1987;3:395-410.

23. Opitz JM, Howe JJ. The Meckel syndrome (dysencephaliaspanchnocystica, the Grüber syndrome) Birth Defects. 1969;2:167-179.

24. Martínez-Medel J, Sanz-Asín O, Amat-Villegas I, Azcona Ruiz B, Cabistany-Esqué AC, De-Marcos EM. Síndrome de Meckel. Diagnóstico prenatal y diagnóstico diferencial. Prog Obstet Ginecol. 2012;55(6):269-273. Disponible en: http://www.elsevier.es/es-revista-progresos-obstetriciaginecologia-151-articulo-sindrome-meckel-diagnosticoprenatal-diagnostico-S0304501312000672\#elsevierltem Bibliografias

25. Al-Belushi M, Al Ibrahim A, Ahmed M, Ahmed B, Khenyab $\mathrm{N}$, Konje JC. A review of Meckel-Gruber syndrome - incidence and outcome in the state of Qatar. J Matern Fetal Neonatal Med. 2015;3:14. http://www.ncbi.nlm.nih.gov/ pubmed/26333300 